\title{
The critical region thermodynamics of some statistical models
}

\author{
E.D.Soldatova, O.M.Galdina \\ Dnipropetrovs'k National University
}

Received July 18, 2005, in final form October 31, 2005

We consider the exactly solvable Ashkin-Teller model and the hard square model, in which both scaling law hypothesis and the universality one are violated. The critical properties of these models have been investigated. The reasons for the violation of the scaling law and universality hypotheses are clarified.

Key words: stability coefficients, violation of the scaling law and the universality hypotheses

PACS: $64.60 . F r$

\section{Preface}

The successes of the modern theory of critical phenomena are connected with the fundamental conclusions regarding the nature of the critical state and the existence of the class of systems, for which both the scaling law hypothesis and the universality one are fulfilled. However, some theses of this theory have not been strictly proved so far. Besides, a special form of Hamiltonian is used. From this point of view the existence of real systems and consistent models contradicting the above-mentioned hypotheses are the problem of great importance. The 6-vertex Lieb model and the hard square model, in which the scaling law hypothesis is violated, as well as Baxter model and Ashkin-Teller one, in which the universality hypothesis is violated, are the models of this kind. The main aim of this paper is to derive thermodynamic properties of Ashkin-Teller model and the hard square model in the vicinity of critical point. We analyze the behaviour of the whole set of stability characteristics (the adiabatic and isodynamic parameters) in the critical region and determine the critical behaviour types for these models based on the thermodynamic method for the one-component system critical state. The method is based on the constructive critical state definition and on the condition of critical state stability. It leads to the existence of four types of critical behaviour [1,2]. We argue the reasons for the 
violation of the scaling law and the universality hypotheses in Ashkin-Teller model as well as in the hard square model.

Let us consider the basic theses of thermodynamic method.

\section{The thermodynamic method for the critical state}

The basic parameters characterizing a thermodynamic system from the standpoint of its stability are the adiabatic parameters (the AP's) $\left(\frac{\partial T}{\partial S}\right)_{x},\left(\frac{\partial T}{\partial x}\right)_{S}$, $\left(\frac{\partial X}{\partial x}\right)_{S}$, and the isodynamic parameters (the IP's) $\left(\frac{\partial T}{\partial S}\right)_{X},\left(\frac{\partial T}{\partial x}\right)_{X},\left(\frac{\partial X}{\partial x}\right)_{T}$. The parameters $\left(\frac{\partial T}{\partial S}\right)_{x}$ and $\left(\frac{\partial X}{\partial x}\right)_{S}$ are called the adiabatic stability coefficients (the ASC's), and the parameters $\left(\frac{\partial T}{\partial S}\right)_{X}$ and $\left(\frac{\partial X}{\partial x}\right)_{T}$ are called the isodynamic stability coefficients (the ISC's) [3,4]. They are related to the fluctuations of the external system parameters (the first and the second Gibbs lemmas), which infinitely increase near the critical point. One can consider the volume $V$, the magnetization $M$ and the electric polarization $\mathcal{P}$ as the generalized thermodynamic variables (the external parameters) $x$. The conjugated thermodynamic forces are the pressure $P$, the electric field strength $E$ and the magnetic field strength $H$.

The most complete critical state definition, which includes both the properties of heterogeneous and homogeneous system (in the subcritical and supercritical region, correspondingly) can be written in the following form $[1,2]$ :

$$
\left\{\begin{array}{c}
\mathrm{d} T=\left(\frac{\partial T}{\partial S}\right)_{x} \mathrm{~d} S+\left(\frac{\partial T}{\partial x}\right)_{S} \mathrm{~d} x=0 \\
\mathrm{~d} X=\left(\frac{\partial X}{\partial S}\right)_{x} \mathrm{~d} S+\left(\frac{\partial X}{\partial x}\right)_{S} \mathrm{~d} x=0
\end{array}, \quad\left(\frac{\partial X}{\partial T}\right)_{\mathrm{c}}=-\frac{\mathrm{d} S}{\mathrm{~d} x}=K_{\mathrm{c}}\right.
$$

where $K_{\mathrm{c}}$ is the critical slope of a phase equilibrium curve. For the existence of non-trivial solutions of (1) the condition

$$
\left[\begin{array}{ll}
\left(\frac{\partial T}{\partial S}\right)_{x} & \left(\frac{\partial T}{\partial x}\right)_{S} \\
\left(\frac{\partial T}{\partial x}\right)_{S} & \left(\frac{\partial X}{\partial x}\right)_{S}
\end{array}\right]=D=\left(\frac{\partial T}{\partial S}\right)_{x}\left(\frac{\partial X}{\partial x}\right)_{S}-\left(\frac{\partial T}{\partial x}\right)_{S}^{2}=0
$$

has to be fulfilled all over the spinodal.

It coincides with the well-known critical state condition $D=0$, where $D$ is the stability determinant $[3,4]$.

The definition (1) describes the critical state by means of the AP's. The critical slope $K_{\mathrm{c}}$ is the solution of a set of homogeneous linear equations (1)

$$
-\frac{\mathrm{d} S}{\mathrm{~d} x}=K_{\mathrm{c}}=\left[\operatorname{sign}\left(\frac{\partial T}{\partial x}\right)_{S}\right]\left(\left(\frac{\partial X}{\partial x}\right)_{S}\left(\frac{\partial T}{\partial S}\right)_{x}^{-1}\right)^{1 / 2}
$$


It is the fundamental characteristic of the critical state and expressed by the ASC's.

This definition combined with the critical state stability conditions [1,2] leads to a variety of critical state manifestations, namely, to the existence of four alternative types of critical behaviour of thermodynamic systems:

1. $\left(\frac{\partial T}{\partial S}\right)_{x} \neq 0,\left(\frac{\partial X}{\partial x}\right)_{S} \neq 0 \Rightarrow\left(\frac{\partial T}{\partial x}\right)_{S} \neq 0, K_{\mathrm{c}} \neq\{0, \infty\}$

2. $\left(\frac{\partial T}{\partial S}\right)_{x}^{x} \neq 0,\left(\frac{\partial X}{\partial x}\right)_{S}=0 \Rightarrow\left(\frac{\partial T}{\partial x}\right)_{S}^{S}=0, K_{\mathrm{c}}=0$

3. $\left(\frac{\partial T}{\partial S}\right)_{x}^{x}=0,\left(\frac{\partial X}{\partial x}\right)_{S} \neq 0 \Rightarrow\left(\frac{\partial T}{\partial x}\right)_{S}^{S}=0, K_{\mathrm{c}}=\infty$

4. $\left(\frac{\partial T}{\partial S}\right)_{x}^{x}=0,\left(\frac{\partial X}{\partial x}\right)_{S}^{S}=0 \Rightarrow\left(\frac{\partial T}{\partial x}\right)_{S}^{S}=0, K_{\mathrm{c}}$ is not defined by (1).

The critical behaviour type of a specific physical system is defined by the value of one ASC and $K_{\mathrm{c}}$. The behaviour of the whole set of stability characteristics (the AP's and IP's) can be determined for each critical type. The fourth type of critical behaviour is the most interesting and the most "fluctuating" one. In this case both the ASC's tend to zero (and, hence, the rest of stability characteristics tend to zero). As a consequence the critical slope is not determined by set (1). In this case it is necessary to consider the differential equations of higher orders. Then the solution is realized through several possibilities $[1,2]$. The case of two or even three phase equilibrium curves converging at the critical point is of special interest. As we demonstrated in [5], the critical point of the ferroelectric Lieb model is of this kind.

\section{The critical properties of Ashkin-Teller model}

J.Ashkin and E.Teller [6] suggested their model as a generalization of Ising model for the four-component system. Each lattice site in this model is occupied by an atom of one of the four kinds: $A, B, C$ or $D$. The interaction energy of two neighbours is equal to $\varepsilon_{0}$ for $A A, B B, C C, D D ; \varepsilon_{1}$ for $A B, C D ; \varepsilon_{2}$ for $A C, B D$ and $\varepsilon_{3}$ for $A D$, $B C$.

The model can be expressed by the Ising spins. Each site $i$ is associated with two spins: $s_{i}$ and $\sigma_{i}$. Let $\left(s_{i}, \sigma_{i}\right)=(+,+),(+,-),(-,+),(-,-)$ if site $i$ is occupied by the atom $A, B, C, D$, correspondingly. Then the interaction energy for the edge $(i, j)$ has the form

$$
\begin{aligned}
\varepsilon(i, j) & =-J s_{i} s_{j}-J^{\prime} \sigma_{i} \sigma_{j}-J_{4} s_{i} \sigma_{i} s_{j} \sigma_{j}-J_{0}, \\
-J & =\left(\varepsilon_{0}+\varepsilon_{1}-\varepsilon_{2}-\varepsilon_{3}\right) / 4,-J^{\prime}=\left(\varepsilon_{0}+\varepsilon_{2}-\varepsilon_{3}-\varepsilon_{1}\right) / 4, \\
-J_{4} & =\left(\varepsilon_{0}+\varepsilon_{3}-\varepsilon_{1}-\varepsilon_{2}\right) / 4,-J_{0}=\left(\varepsilon_{0}+\varepsilon_{1}+\varepsilon_{2}+\varepsilon_{3}\right) / 4 .
\end{aligned}
$$

The partition function is defined by the expression

$$
Z_{A T}=\sum_{s} \sum_{\sigma} \exp \left[-\sum_{(i, j)} \varepsilon(i, j) / k T\right]
$$


where $k$ is Boltzmann constant and $T$ is the temperature. The sum in the exponent runs over all the curves $(i, j)$, which join the neighbouring sites of the lattice; the outer sums are taken over the values of all the spins $s_{1}, s_{2}, s_{3}, \ldots$ and $\sigma_{1}, \sigma_{2}, \sigma_{3}, \ldots$

The dimensionless coefficients of interaction and the Boltzmann weighting coefficients are defined by

$$
\begin{array}{cc}
K=J / k T, & K^{\prime}=J^{\prime} / k T, \\
K_{4}=J_{4} / k T, & K_{0}=J_{0} / k T, \\
\omega_{0}=\exp \left(K+K^{\prime}+K_{4}+K_{0}\right), & \omega_{1}=\exp \left(K-K^{\prime}-K_{4}+K_{0}\right), \\
\omega_{2}=\exp \left(-K+K^{\prime}-K_{4}+K_{0}\right), & \omega_{3}=\exp \left(-K-K^{\prime}+K_{4}+K_{0}\right) .
\end{array}
$$

This consideration is valid for any lattice, not necessarily the plane one. Usually, the square lattice with $N$ sites is analyzed. As it follows from (5), Ashkin-Teller model can be presented as two Ising models on a square lattice ( $s$-model and $\sigma$ model) connected by means of the four-spin interactions.

The model is also similar to the eight-vertex model [7] in the absence of external field with the Hamiltonian

$$
\begin{aligned}
\mathcal{H}= & -\sum_{i=1}^{M} \sum_{j=1}^{N}\left\{J_{v} \sigma_{i j} \sigma_{i, j+1}+J_{h} \sigma_{i j} \sigma_{i+1, j}\right. \\
& \left.+J \sigma_{i, j+1} \sigma_{i+1, j}+J^{\prime} \sigma_{i j} \sigma_{i+1, j+1}+J^{\prime \prime} \sigma_{i j} \sigma_{i, j+1} \sigma_{i+1, j}\right\},
\end{aligned}
$$

in which $J_{v}=J_{h}=0$. However, the lattice geometry is different in these two cases. The spins of the eight-vertex model are located at different sites, whereas the spins of both kinds $s_{i}$ and $\sigma_{i}$ are located at the same site $i$ in Ashkin-Teller model.

The critical exponents for Ashkin-Teller model can be written in the form:

$$
\begin{aligned}
\alpha=\frac{2-2 y}{3-2 y}, & \beta_{m}=\frac{2-y}{24-16 y}, & \gamma_{m}=\frac{14-7 y}{12-8 y}, \quad \delta_{m}=15 ; \\
\beta_{e}=\frac{1}{12-8 y}, & \gamma_{e}=\frac{7-4 y}{6-4 y}, & \delta_{e}=15-8 y,
\end{aligned}
$$

where $y$ is the interaction parameter which is related to the Baxter model interaction parameter [7] in the following way $y=2 \mu / \pi, 0<\mu<2 \pi / 3$.

Therefore, there are two sets of critical exponents: the "magnetic" exponents, corresponding to the field $-H \sum \sigma_{i}$, and the "electric" exponents, corresponding to the field $-E \sum \sigma_{i} s_{i}$. Here we use indices $m$ and $e$ for the "magnetic" and "electric" exponents. So, $\beta_{m}$ is the critical exponent for the order parameter $\left\langle\sigma_{1}\right\rangle$ and $\beta_{e}$ is the critical exponent for the order parameter $\left\langle\sigma_{1} s_{1}\right\rangle$.

Both Ashkin-Teller model and Baxter model disobey the universality principle, since their critical exponents depend on the interaction parameter $y$ continuously.

Thus, the isodynamic stability coefficients for the "magnetic" Ashkin-Teller model have the following asymptotic form

$$
\left(\frac{\partial T}{\partial S}\right)_{H} \sim(-t)^{\frac{2-2 y}{3-2 y}}, \quad\left(\frac{\partial H}{\partial M}\right)_{T} \sim(-t)^{\frac{14-7 y}{12-8 y}}
$$


where $t=\left(T-T_{\mathrm{c}}\right) / T_{\mathrm{c}}$. In the absence of the external field (this case is the subject of consideration here) the isodynamic and the adiabatic parameters coincide. Hence, the asymptotic expressions for adiabatic parameters are as follows:

$$
\left(\frac{\partial T}{\partial S}\right)_{M} \sim(-t)^{\frac{2-2 y}{3-2 y}}, \quad\left(\frac{\partial H}{\partial M}\right)_{S} \sim(-t)^{\frac{14-7 y}{12-8 y}}, \quad\left(\frac{\partial T}{\partial M}\right)_{S} \sim(-t)^{\frac{26-17 y}{24-16 y}} .
$$

Let us analyze the ASC's behaviour. When $1<y<4 / 3$, that corresponds to $\pi / 2<\mu<2 \pi / 3$, the critical exponent $\alpha$ becomes negative, the exponent $\gamma$ is positive, i.e. the second type of the critical behaviour with the critical slope value $K_{\mathrm{c}}=0$ is realized. All the inverse susceptibilities (the adiabatic and isothermic) as well as the parameters $\left(\frac{\partial T}{\partial M}\right)_{S}$ and $\left(\frac{\partial T}{\partial M}\right)_{H}$ tend to zero at the critical point, and the thermic stability coefficients $\left(\frac{\partial T}{\partial S}\right)_{M}$ and $\left(\frac{\partial T}{\partial S}\right)_{H}$ are finite. At $y=1$ the critical exponents are as follows: $\alpha=0, \beta=1 / 8, \gamma=7 / 4$. This coincides with the 2D Ising model results [9] and corresponds to the fourth type of critical behaviour with $K_{\mathrm{c}}=0$. At $0 \leqslant y<1(0 \leqslant \mu<\pi / 2)$ the exponent $\alpha$ becomes positive and $\alpha<\gamma$ - the fourth type of critical behaviour with $K_{\mathrm{c}}=0$ is fulfilled. When $y=0$, we have $\alpha=2 / 3, \beta=1 / 12, \gamma=7 / 6$, i.e. all the critical exponents coincide with the critical exponents of the three-spin model [10] realizing the fourth type of critical behaviour with $K_{\mathrm{c}}=0$.

For the "electric" Ashkin-Teller model the isodynamic stability coefficients are as follows:

$$
\left(\frac{\partial T}{\partial S}\right)_{E} \sim(-t)^{\frac{2-2 y}{3-2 y}}, \quad\left(\frac{\partial E}{\partial \mathcal{P}}\right)_{T} \sim(-t)^{\frac{7-4 y}{6-4 y}}
$$

and the adiabatic parameters are

$$
\left(\frac{\partial T}{\partial S}\right)_{\mathcal{P}} \sim(-t)^{\frac{2-2 y}{3-2 y}}, \quad\left(\frac{\partial E}{\partial \mathcal{P}}\right)_{S} \sim(-t)^{\frac{7-4 y}{6-4 y}}, \quad\left(\frac{\partial T}{\partial \mathcal{P}}\right)_{S} \sim(-t)^{\frac{13-8 y}{12-8 y}} .
$$

At the values of the interaction parameter $1 \leqslant y<4 / 3$, which corresponds to $\pi / 2 \leqslant \mu<2 \pi / 3$, the second type of critical behaviour is also fulfilled. When $0 \leqslant y<1(0 \leqslant \mu<\pi / 2)$, the critical exponent $\alpha$ is always less than the critical exponent $\gamma$, and the fourth type of critical behaviour with critical slope $K_{\mathrm{c}}=0$ is realized. The case $y=0$, as above, corresponds to the three-spin model.

Consequently, the performed analysis shows, that the violation of the universality hypothesis in Ashkin-Teller model is concerned with the dependence of the critical behaviour type on the interaction parameter $y$. The second or the fourth type of critical behaviour is possible, which is characterized by the different order of fluctuation growth at the critical point. 


\section{The features of critical behaviour of the hard square model}

One of the exactly solvable models of statistical physics is the hard hexagon model [8]. It is the two-dimensional lattice model of hard molecules (the molecules, which do not overlap). In this model the particles are situated at sites of the triangular lattice in such a way, that any two of them are not allowed to be located both at the same site or at neighbouring sites. Let us imagine that each particle is situated at the centre of hexagon, which covers the six neighbouring edges of the triangular lattice. Then all these hexagons do not overlap. That could explain the name of this model.

Choosing the lattice with the edges of the right-angled triangular form, we obtain a more general model, in which the nearest neighbour sites cannot be occupied and the particles at the diagonals of square edges interact. Each spin $\sigma_{i}$ can take the values 1 or 0 . This model is called a hard square model [8]. It is the limiting case of the lattice gas with the interaction between the nearest neighbours. There is a phase transition from the homogeneous liquid state at small activities $z$ to the inhomogeneous solid state at large activities. The partition function can be expressed as

$$
Z=\sum_{\sigma} z^{\sigma_{1}+\cdots+\sigma_{N}} \prod_{(i, j)}\left(1-\sigma_{i} \sigma_{j}\right)
$$

where the product is carried out all over the edges $(i, j)$ of the lattice, and the summation is performed all over the values $(0,1)$ for all quantities $\sigma_{1}, \ldots, \sigma_{N}$.

The critical exponents in this models are as follows:

$$
\alpha^{\prime}=-\frac{1}{2}, \quad \alpha=\frac{3}{4}, \quad \beta=\frac{1}{4},
$$

i.e. the critical exponent for the heat capacity is different in the subcritical and supercritical region $\alpha \neq \alpha^{\prime}$. It means that the scaling law hypothesis is violated in the model. We have already dealt with such a situation in Lieb model [5]. The stability coefficients in the subcritical region are

$$
\left(\frac{\partial T}{\partial S}\right)_{V} \sim(-t)^{-1 / 2}, \quad\left(-\frac{\partial P}{\partial V}\right)_{S}=\mathrm{const}
$$

i.e.

$$
\left(\frac{\partial T}{\partial S}\right)_{V}=0, \quad\left(-\frac{\partial P}{\partial V}\right)_{S}=0
$$

The critical slope is $K_{\mathrm{c}} \neq\{0, \infty\}$, so, the first type of critical behaviour is realized. In the supercritical region

$$
\begin{aligned}
& \left(\frac{\partial T}{\partial S}\right)_{V} \sim(-t)^{3 / 4}, & & \left(-\frac{\partial P}{\partial V}\right)_{S} \sim(-t)^{3 / 4}, \\
\text { i.e. } & \left(\frac{\partial T}{\partial S}\right)_{V} \neq 0, & & \left(-\frac{\partial P}{\partial V}\right)_{S} \neq 0,
\end{aligned}
$$


so, the fourth type of critical behaviour with the critical slope of the phase equilibrium curve $K_{\mathrm{c}} \neq\{0, \infty\}$ is fulfilled.

These are the distinct types of critical behaviour in the sense of fluctuation nature - the second type is characterized by the minimal order of the energy and compressibility fluctuation growth. For the fourth type all the fluctuations are extremely large. The reason for the violation of the scaling law hypothesis in the hard square models concerns this very fact.

\section{Conclusions}

The paper considers the thermodynamic stability of Ashkin-Teller model and the hard square model using the method $[1,2]$. The asymptotic expressions for the whole set of stability characteristics are determined and the reasons for the violation of the scaling law and the universality hypotheses in given models are clarified. In the hard square model, which can be applied to describing the critical behaviour of a liquid, the first type of critical behaviour is realized in the subcritical region, whereas the fourth type is fulfilled in the supercritical region. The violation of the scaling law hypothesis can be explained just through the difference of the behaviour types.

In Ashkin-Teller model which describes the critical behaviour of (anti)ferromagnets and (anti)ferroelectrics the second and fourth type of critical behaviour takes place. The reason for the violation of the universality hypothesis is that each of the mentioned types is related to the value of interaction parameter. It is interesting to emphasize that in both models the violation of one hypothesis does not spoil another one. Therefore, the capabilities of the thermodynamic method for a one-component system critical state are illustrated using the above-mentioned models. The global reasons are revealed for the violation of the scaling law and the universality hypotheses concerning the variety of the critical state manifestations.

\section{References}

1. Soldatova E.D. Thermodynamic Stability in Critical State Region. Thesis for a Doctor's degree (Physics and Mathematics). Kyiv, KSU, 1991 (in Russian).

2. Soldatova E.D., Cond. Matt. Phys., 1999, 2, No. 4(20), 603-616.

3. Semenchenko V.K. Selected Chapters of Theoretical Physics. Prosveschenie, Moscow, 1966.

4. Semenchenko V.K., Crystallography, 1964, 9, No. 5, 611-621 (in Russian).

5. Soldatova E.D., Galdina O.M., Ukr. J. Phys., 2004, 49, No. 11, 1122-1126.

6. Ashkin J., Teller E., Phys. Rev., 1943, 64, No. 5-6, 178-184.

7. Baxter R.J., Stud. Appl. Math, 1970, L, No. 1, 51-69.

8. Baxter R.J. Exactly Solvable Models in Statistical Mechanics. Academic Press, London, 1982.

9. Stanley H.E. Introduction to Phase Transitions and Critical Phenomena. Clarendon Press, Oxford, 1971.

10. Baxter R.J., Enting I.G., J. Phys. A: Math. Gen., 1976, 9, L149-L151. 


\section{Термодинаміка критичної області деяких статистичних моделей}

\section{Є.Д.Солдатова, О.М.Галдіна}

Дніпропетровський національний університет

Отримано 18 липня 2005 р., в остаточному вигляді 31 жовтня 2005 р.

Неабиякий інтерес для сучасної теорії критичних явищ становлять послідовні точно розв'язувані моделі статистичної механіки, в яких порушуються гіпотези подібності й універсальності. До них відносять модель Ешкіна-Теллера і модель жорстких квадратів. В даній роботі досліджено їх критичні властивості. Пояснюються причини порушення гіпотез подібності й універсальності.

Ключові слова: коефіцієнти стійкості, порушення гіпотез подібності й універсальності

PACS: $64.60 . F r$ 